\title{
Enhancers in the Peril lincRNA locus regulate distant but not local genes
}

\author{
Abigail F. Groff ${ }^{1,2}$, A. Rasim Barutcu ${ }^{1,3}$, Jordan P. Lewandowski ${ }^{1}$ and John L. Rinn ${ }^{1,4^{*}}$
}

\begin{abstract}
Background: Recently, it has become clear that some promoters function as long-range regulators of gene expression. However, direct and quantitative assessment of enhancer activity at long intergenic noncoding RNA (lincRNA) or mRNA gene bodies has not been performed. To unbiasedly assess the enhancer capacity across lincRNA and mRNA loci, we performed a massively parallel reporter assay (MPRA) on six lincRNA loci and their closest protein-coding neighbors.

Results: For both gene classes, we find significantly more MPRA activity in promoter regions than in gene bodies. However, three lincRNA loci, Lincp21, LincEnc1, and Peril, and one mRNA locus, Morc2a, display significant enhancer activity within their gene bodies. We hypothesize that such peaks may mark long-range enhancers, and test this in vivo using RNA sequencing from a knockout mouse model and high-throughput chromosome conformation capture (Hi-C). We find that ablation of a high-activity MPRA peak in the Peril gene body leads to consistent dysregulation of Mcccl and Exosc9 in the neighboring topologically associated domain (TAD). This occurs irrespective of Peril lincRNA expression, demonstrating this regulation is DNA-dependent. Hi-C confirms long-range contacts with the neighboring $T A D$, and these interactions are altered upon Peril knockout. Surprisingly, we do not observe consistent regulation of genes within the local TAD. Together, these data suggest a long-range enhancer-like function for the Peril gene body.

Conclusions: A multi-faceted approach combining high-throughput enhancer discovery with genetic models can connect enhancers to their gene targets and provides evidence of inter-TAD gene regulation.
\end{abstract}

Keywords: lincRNA, Mouse models, MPRA, Cis-regulation, Enhancer

\section{Background}

While a number of lincRNAs function by RNA-based mechanisms [1], recently, it has become clear that lincRNA loci also harbor functional DNA regulatory elements [2-7]. These DNA regions may account for the majority of the regulatory capacity of a lincRNA locus $[3,4]$. To date, most efforts to characterize DNA elements within lincRNA loci have only focused on promoter regions [8-12] or predictions from genome-wide binding of p300 or H3K4me1 and H3K4me3 marks [13, 14]. The former approach has highlighted the crosstalk between neighboring lincRNA and mRNA promoters, suggesting that promoters of both mRNAs and lincRNAs can

\footnotetext{
*Correspondence: john.rinn@colorado.edu

'Department of Stem Cell and Regenerative Biology, Harvard University, Cambridge, MA 02138, USA

${ }^{4}$ Department of Biochemistry, BioFrontiers, University of Colorado Boulder, Boulder, CO 80301, USA

Full list of author information is available at the end of the article
}

function as long-range enhancers to regulate transcription [15]. Similarly, mRNA loci have been shown to contain DNA enhancers in introns, UTRs, and even exons [12, 13, 16-20]. These studies unearth a complex regulatory landscape in both mRNA and lincRNA loci that requires multiple experimental and analytical approaches to properly decouple the contributions of DNA, transcription, RNA, and protein to regulation of gene expression. However, despite the success of these studies, our understanding of the contributions of potential enhancer elements lying within gene bodies to this complex regulatory landscape remains incomplete.

To disentangle the contributions of RNA and DNA at a set of developmentally regulated lincRNA loci, we previously generated 18 lincRNA knockout mouse models $[21,57]$. In these models, the gene body is replaced by a $L a c Z$ reporter that is downstream of the endogenous

(c) The Author(s). 2018 Open Access This article is distributed under the terms of the Creative Commons Attribution 4.0 International License (http://creativecommons.org/licenses/by/4.0/), which permits unrestricted use, distribution, and reproduction in any medium, provided you give appropriate credit to the original author(s) and the source, provide a link to the Creative Commons license, and indicate if changes were made. The Creative Commons Public Domain Dedication waiver (http://creativecommons.org/publicdomain/zero/1.0/) applies to the data made available in this article, unless otherwise stated. 
promoter [21]. Thus, regulation of neighboring genes (cis-like regulation) in tissues where the reporter is not expressed implicates DNA and not the act of transcription or RNA product as the regulatory driver. This is because when a region is transcriptionally silent, neither transcription nor an RNA product is present to influence gene expression. Thus, such models allow for the direct detection of DNA-based regulatory elements.

Using these mouse models, we identified several lincRNA loci, including Crnde, LincEnc1, Lincp21, and Tug1, which appear to function as cis-regulators controlling expression in their genomic neighborhoods $[6,22]$. However, it remains unclear if these regulatory roles are due to RNA-mediated interactions or underlying DNA. To directly interrogate these loci for DNA-based enhancer activity, we performed a massively parallel reporter assay (MPRA) using oligonucleotides that tile the promoters and gene bodies of six lincRNA and mRNA gene pairs. These loci include the four potential cis-acting lincRNA loci (Crnde, LincEnc1, Lincp21, and Tug1) and two lincRNA loci with undetermined regulatory function (Peril and Fendrr), as well as their closest protein-coding neighbors [6, 21]. Using this method, we show that the majority of MPRA-based enhancer activity in these loci arises from the promoter regions (mRNAs and lincRNAs alike). However, we also identify four loci, Morc2a (mRNA), Lincp21, LincEnc1, and Peril (lincRNAs), which harbor high MPRA activity in their gene bodies, consistent with promoter-independent enhancer activity.

To determine whether gene body MPRA peaks indicate enhancer activity in vivo, we used a previously generated Peril knockout mouse model to dissect the DNA regulatory roles of the gene body, excluding the promoter, at this locus. We focused on Peril because it has one of the highest gene body MPRA peaks and because it overlaps with a super-enhancer for Sox2, which is a critical stem cell regulator [23-25]. Surprisingly, upon deletion of Peril, we do not observe significant dysregulation of nearby genes (e.g., Sox2). Instead, we find that two genes, Exosc9 and Mccc1, which are distally located $(\sim 1.5 \mathrm{Mb}$ away) from the Peril locus in the neighboring TAD, are significantly downregulated in all four tissues examined. The downregulation of these genes occurred whether the Peril region was transcribed or silent. Moreover, comparison of high-throughput chromosome capture (Hi-C) data from wild-type and Peril knockout murine embryonic stem cells (mESCs) revealed alterations in physical long-range interactions between the Peril region and the TAD containing Mccc1 and Exosc9 genes. Taken together, our results quantify the presence of gene body DNA regulatory elements within lincRNA loci and identify their corresponding candidate target genes.

\section{Results}

\section{MPRA to interrogate locus activity}

To determine whether lincRNA loci might contain enhancer activity in their gene bodies, we performed an MPRA screen of six lincRNAs: Crnde, Fendrr, LincEnc1, Lincp21, Peril, and Tug1. To directly compare lincRNA with mRNA loci, we also included the nearest proteincoding gene to each respective lincRNA: Irx5, Foxf1a, Enc1, Cdkn1a, Sox2, and Morc2a, respectively (Fig. 1a). This approach allowed us to precisely interrogate the enhancer capacity of the DNA sequence, offering a quantitative advantage over ChIP-based methods which are unable to measure the transcriptional activating potential of DNA.

The MPRA method relies on the synthesis of thousands of oligos spanning a genomic region of interest, and can be used to systematically dissect the location of regulatory elements $[3,26-28]$. We generated $\sim 22,000$ distinct oligos tiling the 12 loci and their promoters (defined as $1 \mathrm{~kb}$ upstream of each transcriptional start site (TSS)) (Fig. 1a, Additional files 1 and 2). Each oligo is comprised of 90 nucleotides (nt) of genomic sequence, two restriction enzyme sites, a unique 10nt barcode sequence, and flanking universal primer sites. The oligo pool is then cloned into a minimal vector, and a GFP reporter is inserted. This results in a barcode-tagged GFP that is transcribed in transiently transfected cells and can be used as a readout of activity (Fig. 1b). To ensure high redundancy in our dataset, we tiled each locus in 50nt intervals and have designed the pool such that every $90 \mathrm{nt}$ genomic element is represented by five unique barcodes.

\section{MPRA activity is enriched in promoters}

We performed five replicate transfections in $\mathrm{C} 2 \mathrm{C} 12$ cells, a mouse myoblast cell line in which to the genes of interest have varied expression status (Fig. 1c). As a quality control measure, we assessed replicate correlation between RNA samples and between DNA control libraries (Additional file 3: Figure S1). Normalized replicate samples correlated well with one another $\left(R^{2}>0.99\right.$, Additional file 3: Figure S1), while exhibiting clear signal with respect to DNA input control $\left(R^{2} \sim 0.8544\right.$, Fig. 1d). To evaluate the dynamic range of the MPRA, we included tiles across a promoter known to be highly active in this cell type (cytomegalovirus) and scrambled copies of this promoter. Tiles corresponding to the scrambled promoter exhibited significantly less activity than those corresponding to the proper sequence, indicating the specificity of the assay (Fig. 1e). Together, these results highlight the quality and reproducibility of our MPRA data.

In order to define significantly active MPRA regions, we leveraged our tiling design using a sliding window statistic (see the "Methods" section). Significant regions 
A

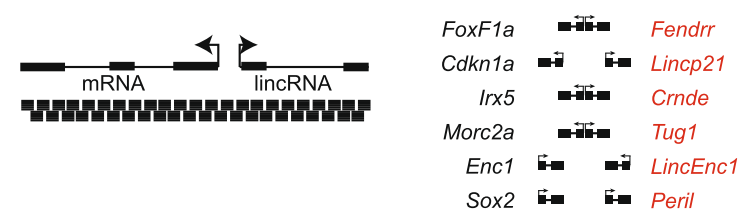

B

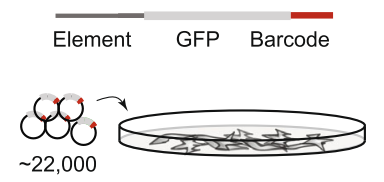

E

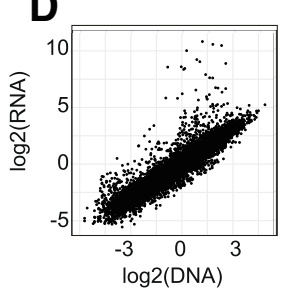

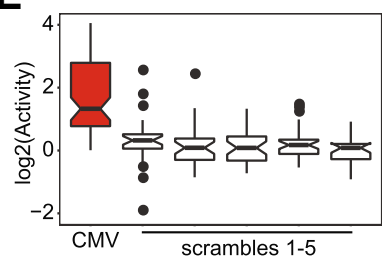

$\mathbf{F}$

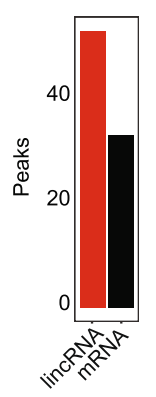

G

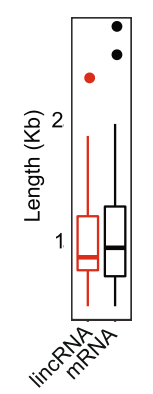

H

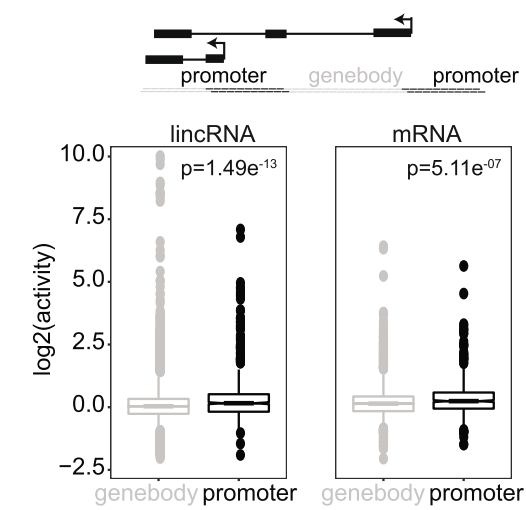

Fig. 1 MPRA in C2C12 cells identifies sequences with differential enhancer activity. a Summary of MPRA pool design. Cis-acting lincRNAs (right) and their closest protein-coding neighbor (left) are redundantly tiled by 90-bp windows starting every $50 \mathrm{bp}$. $\mathbf{b}$ Core oligo design and experimental overview. Actual oligos flanked by universal primer sites for amplification. Element corresponds to 90-bp genomic sequence, barcode is a 10 nt unique identifying tag, and GFP with minimal promoter is inserted after restriction enzyme digestion. c C2C12 expression (TPM) for each gene in the MPRA pool. Red indicates lincRNA, and gray indicates mRNA. $\mathbf{d}$ Median sample scatter plot of C2C12 RNA to DNA input control barcode counts (median across replicates used for each barcode, normalized for sequencing depth). e Boxplots of relative signal originating from CMV-promoter-tiling barcodes or oligos tiling five different scrambled versions of the same sequence. $Y$-axis shows $\log _{2}$ (activity), i.e., $\log _{2}$ (normed RNA counts/normed DNA counts). f Total number of significant regions identified for lincRNAs (red) or mRNAs (gray). $\mathbf{g}$ Size distribution of significant regions identified for lincRNAs (red) or mRNAs (gray). $\mathbf{h}$ Boxplot of gene body- or promoter-originating oligos from lincRNA loci. The gene body is represented in gray, and the promoter is represented in black. Right, same for mRNA loci. Top illustration: Scheme of TSS-based oligo partitioning. All oligos 1000 bp upstream of a TSS are labeled "promoter" (in black), and the remaining oligos are considered "gene body" (in gray)

were calculated using a bootstrapped $p$ value (BH-corrected $p<0.01$, FDR $1 \%)$. Using this threshold, we found 45 unique peaks in our lincRNA loci and 28 peaks in our mRNA loci, highlighting that both types of loci can contain enhancers (Fig. 1f). Average peak size for lincRNA loci and mRNA loci was $1046 \mathrm{bp}$ and $1127 \mathrm{bp}$, respectively (Fig. 1g).

Since many of the active MPRA regions occur near TSSs, we sought to compare the activity distribution of oligos in promoter regions with those in the gene bodies. To do this, we segmented the data by proximity to TSSs. Each oligo was labeled as either promoter-specific (within $1 \mathrm{~kb}$ upstream of a TSS) or gene body-specific (remaining oligos, Fig. 1h). We found that for both lincRNAs and mRNAs, oligos in promoter regions generated significantly more activity than those in gene bodies $(p<1 \mathrm{e}-6$, KolmogorovSmirnov test, Fig. 1h). This finding is consistent with our peak-based observation, with the known functional modalities of classical promoters, and with the role of promoters in long distance gene regulation $[10,15]$. Taken together, our results demonstrate that elements within lincRNA and mRNA promoters alike contribute to cis-regulatory activity more so than their gene body counterparts.

\section{MPRA activity in gene bodies identifies candidate enhancer loci}

While the majority of activity lies in promoter regions, 9 of 12 loci showed significant gene body activity (Fig. 2, Additional file 3: Figure S2). We highlight six loci, three lincRNAs and three mRNAs, demonstrating a range of 

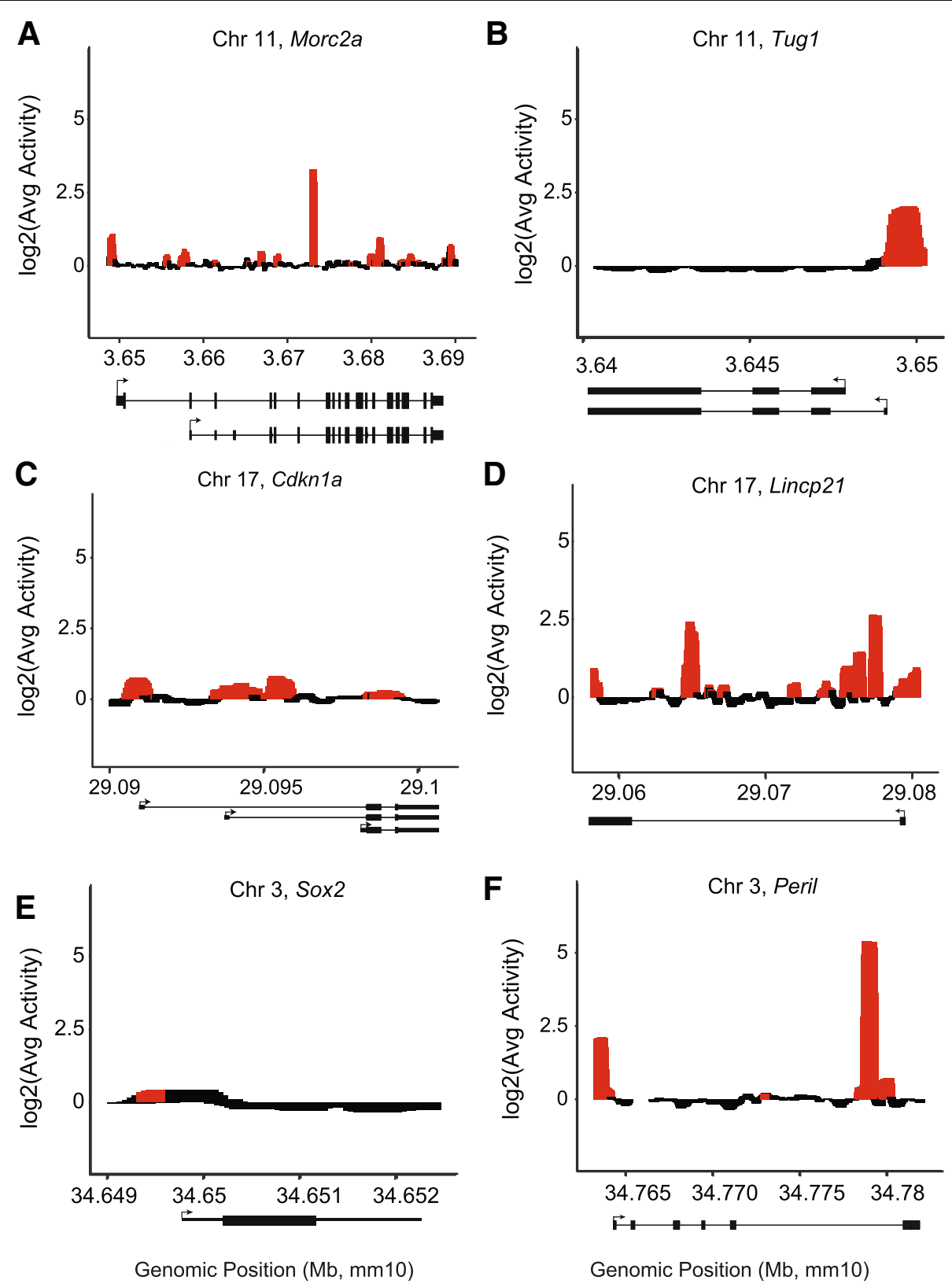

Fig. 2 MPRA reveals enhancer activity and motifs in lincRNA loci. Smoothed signal plot for a protein-coding locus Morc2a and neighboring lincRNA Tugl (b). Red indicates significantly activated regions calculated with a window size of 500 bp and slide of $50 \mathrm{bp}$ (see the "Methods" section). Genomic position (in Mb, mm10) across the $X$-axis, with gene structures indicated below. Y-axis represents $\log _{2}$ (activity). c-f $\operatorname{Same}$ as $\mathbf{a}$ and $\mathbf{b}$ for the $C d k n 1 a$, Lincp21, Sox2, and Peril loci

MPRA activity profiles (Fig. 2). The protein-coding gene Morc $2 a$ contains 16 peaks (Fig. 2a), a finding consistent with the literature on intronic enhancers in mRNA loci [13, 16-20]. The lincRNA Tug1 shares a promoter with Morc $2 a$, but has a dramatically different enhancer profile. While its promoter is active, there are no peaks of activity across its gene body (Fig. 2b). The protein-coding gene Cdkn1a contains four regions of enhancer activity (Fig. 2c), two of which are near TSSs. The neighboring Lincp 21 locus also contains enhancer activity in both its promoter and gene body (Fig. 2d). The protein-coding gene Sox2, which is not expressed in $\mathrm{C} 2 \mathrm{C} 12$ cells, has minimal activity in its promoter and no activity across its gene body (Fig. 2e). Interestingly, however, the lincRNA Peril, which neighbors Sox2, is also not expressed, but showed significant enhancer activity in our MPRA data (Fig. 2f).

In total, four genes, Morc2a, Lincp21, LincEnc1, and Peril, contain high-activity peaks in their gene bodies 
(Log2FoldChange(Ratio) > 2). Morc2a has one 2019-bp high-activity region located in the middle of its gene body (Fig. 2a), while Lincp 21 contains two highly active gene body regions: one 1050-bp region near the $5^{\prime}$ end of the intron and one 1308-bp region near the $3^{\prime}$ end (Fig. 2d). LincEnc1 contains one 1508-bp highly active region in its long central intron (Additional file 3: Figure S2F), while Peril contains one 2361-bp highly active gene body region located near the 3 ' end of its last intron (Fig. 2f). Lincp 21 has already been established as an in vivo enhancer locus [3], and so we chose to further investigate if the presence of a high-activity MPRA peak in the gene body would also indicate in vivo DNA-based enhancer activity in another locus. We chose Peril for further analysis because it has one of the highest MPRA peaks, has been highlighted in previous studies $[6,21]$, and is of broad interest due to its association with the stem cell factor Sox2 [23, 25].

\section{Enhancer candidate locus Peril regulates distal gene targets}

Peril stands out not only because it contains one of the strongest MPRA peaks, but also because of its genomic location. It lies $110 \mathrm{~kb}$ downstream of Sox 2 on murine chromosome 3 and overlaps with a super-enhancer known to regulate Sox2 in mESC (Additional file 3: Figure S3A) [23, 25]. Using an unbiased approach to identify potential cis-like regulatory effects in vivo, we investigated the potential targets of the Peril locus. To do so, we used our Peril knockout mouse, in which approximately $14.5 \mathrm{~kb}$ of the Peril locus was replaced with a LacZ reporter (Fig. 3a, [21]). Importantly, this model removes most of the Peril gene body, including the high-activity MPRA region, while leaving the promoter and super-enhancer regions intact, allowing us to assess the functionality of the gene body region independently of the promoter and super-enhancer. Using this system, observation of cis-like regulatory events in the absence of Peril expression would strongly suggest that this dysregulation is due to loss of the DNA.

To determine cis-regulatory roles of Peril DNA, we performed RNA sequencing on wild-type and Peril knockout embryonic (E14.5) murine tissues and embryonic stem cells (Fig. 3b-d). In total, we sequenced three replicates of wild-type (WT) and knockout (KO) murine embryonic stem cells (mESC) and E14.5 kidney, and two replicates of ganglionic eminence and olfactory bulb (GE/OB). Each library was sequenced to an average depth of $\sim 158$ million reads, for a total of $\sim 2.5$ billion reads (Fig. $3 \mathrm{~b}-\mathrm{d}$ ). We also included three Peril WT and KO replicates of whole brain RNA sequencing that were previously published [6].

First, we wanted to determine the expression level of both Peril and the LacZ reporter in each tissue. We found that Peril is transcriptionally silent in the brain,
$\mathrm{GE} / \mathrm{OB}$, and kidney, but active in $\mathrm{mESC}$, while $L a c Z$ is active in the brain, GE/OB, and mESC, but silent in the kidney (Fig. 3b, c). Next, we sought to identify any changes in gene expression between Peril WT and KO tissues. For each tissue, we performed differential gene expression analysis and found significant perturbations in gene expression $(p<0.05$, DESeq alpha $=0.05$, Fig. $3 \mathrm{~d}$, see the "Methods" section). The most striking observation was the effect on gene expression in the developing kidney, where the Peril region is transcriptionally silent in both the wild-type and knockout (Fig. 3d). In the kidney, we found 76 genes significantly dysregulated, 3 of which are within a $4-\mathrm{Mb}$ region surrounding Peril. The proximity of the 3 dysregulated genes to the Peril locus suggests that these may be candidate targets of the Peril gene body enhancer (Fig. 3e).

To identify potential targets of the Peril locus, we queried the differentially expressed genes for every tissue, independent of the expression status of Peril or the LacZ reporter. Across all four tissues in our study, we identified only two genes that are consistently and significantly downregulated upon Peril deletion, and validated their expression reduction by qRT-PCR (Additional file 3: Figure S3B-C). These two genes are Mccc1 and Exosc9 and are located approximately $1.2 \mathrm{Mb}$ and $1.8 \mathrm{Mb}$ downstream of the Peril locus, respectively (Fig. 3e, Additional file 3: Figure S3B-C). Underscoring the potential importance of the Peril DNA enhancer element, Exosc9 exhibits a prenatal lethal phenotype, consistent with an earlier observation of Peril knockout [21]. The physical proximity of these genes to the deleted Peril region suggests a potential long-range DNA-based regulatory function in the Peril locus.

While there are a number of mechanisms that can influence transcript level, we hypothesized that DNA elements within the Peril locus influence gene expression of Mccc1 and Exosc9, based on our RNA-seq and MPRA data. To gain further insight, we performed a Pol II chromatin immunoprecipitation (ChIP)-qPCR experiment in Peril WT and KO mESC. We designed multiple sets of primers targeting the promoter regions of both candidate target genes and performed qPCR on Pol II-bound chromatin fragments. Interestingly, we found Pol II to be significantly depleted at both the $M c c c 1$ and Exosc9 promoters in knockout mESC (Additional file 3: Figure S3D). These data suggest that enhancers within the Peril locus can regulate gene targets at a long range.

To identify which transcriptional regulators may drive the enhancer signal in the Peril high-activity gene body peak, we used the ChIP-atlas peak browser to identify any significant published transcription factor ChIP peaks in this region. We found evidence for binding of Duxbl1 in $\mathrm{C} 2 \mathrm{C} 12 \mathrm{~s}$, as well as multiple factors in mESC. These include Rad21 (part of the cohesin complex) and CTCF 


\section{A}
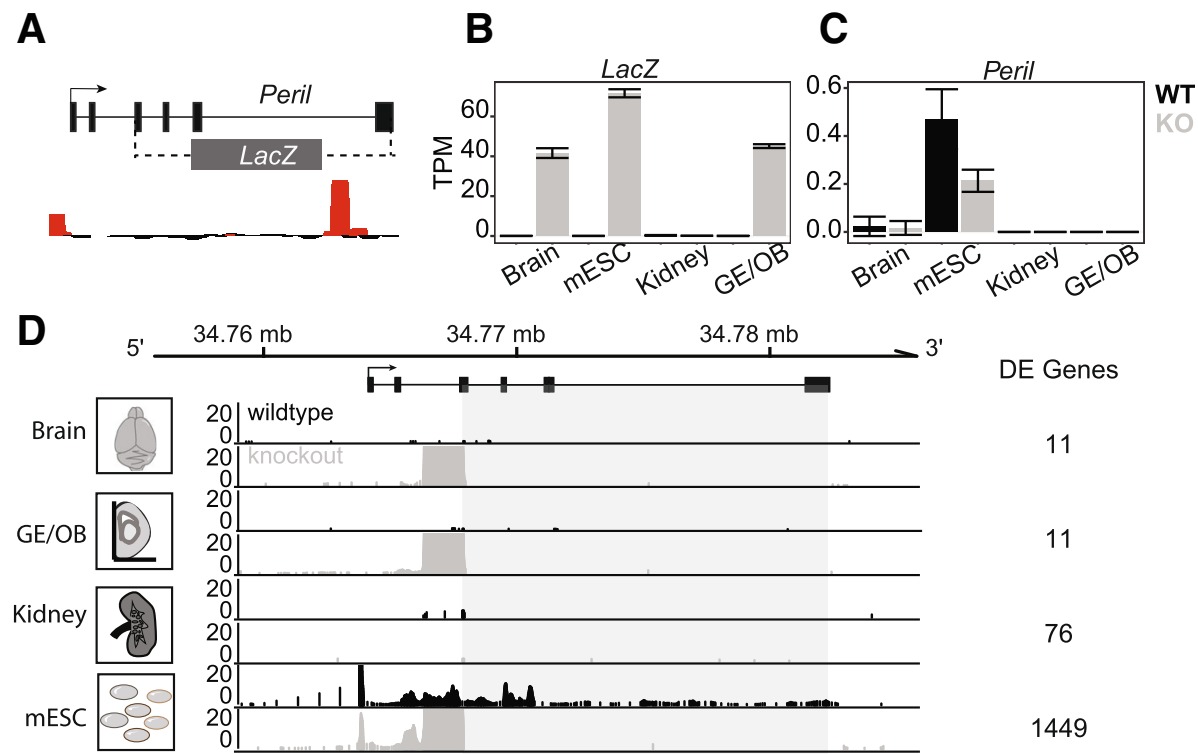

$34.76 \mathrm{mb}$
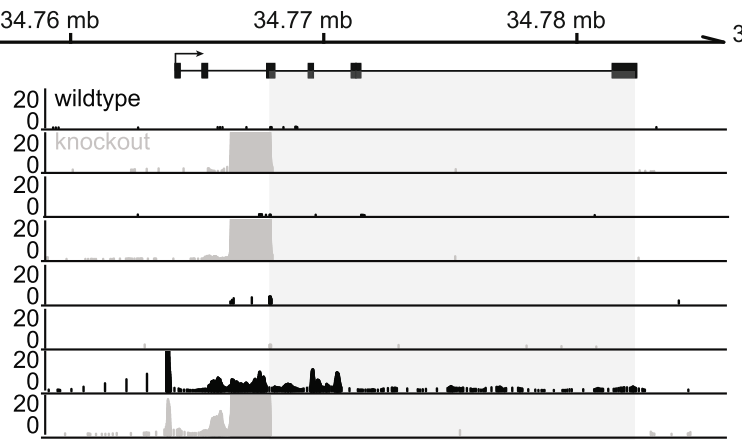

DE Genes

E
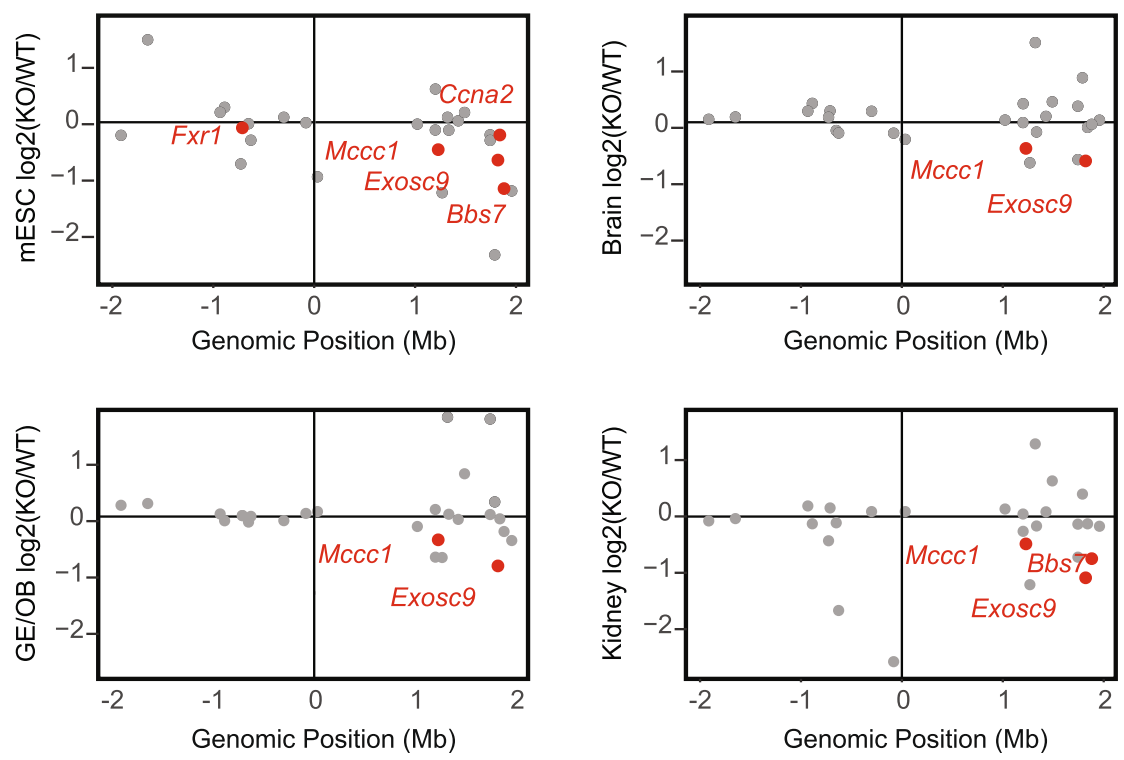

Fig. 3 In vivo RNA sequencing validates DNA-based enhancer in Peril locus. a Peril locus schematic showing knockout region (dotted lines) relative to MPRA peaks (below). b Expression of LacZ and $\mathbf{c}$ Peril in TPMs. WT in black, KO in gray. Error bars indicate 1 standard deviation from the mean. $\mathbf{d}$ Read pileup tracks across the Peril locus. Gray box indicates KO region. WT in black, KO in gray. Tissue type indicated on the left. Number of dysregulated genes in WT-V-KO tissue analysis indicated on the right. e Cis plot of the 4-Mb region surrounding Peril (mm10) for each tissue. Peril locus lies at the origin. Y-axis represents $\log _{2}(\mathrm{KO} / \mathrm{NT}), X$-axis represents genomic position relative to Peril (Mb). Each dot represents a gene expressed in this tissue. Red indicates significantly differential expression between WT and KO. Genes of interest are emphasized

at the very beginning or immediately before the highactivity peak, as well as binding of Rxra (a retinoidresponsive factor also involved in looping), Nr4a1, and Fgfr1 within the peak (Additional file 3: Figure S3E, [29]). A noteworthy limitation of this approach is that ChIP peaks in an enhancer region are limited to which particular factors have been immunoprecipitated in relevant tissues or cells. To circumvent this limitation, we performed motif analysis of our MPRA peak region. While motif analysis is limited by a priori knowledge of specific transcription factor motifs and can be confounded by motif simplicity or redundancy, we can use this analysis to identify candidate factors within our enhancer regions. We downloaded the entire set of motifs available through the Jaspar database (vertebrate collection, [30]), and filtered these for average 
expression greater than or equal to 1TPM in the tissues and cells we sequenced. We then queried the sequence of the high-activity peak in the Peril gene body for matches to any of the expressed transcription factors on a per-sample-type basis using FIMO, a motif-finding tool publically available through the meme suite [31]. We filtered sequence matches for those with a $q$ value $<0.05$ and plotted the common set of expressed hits (Additional file 3: Figure S3F). From this analysis, we find factors that may bind and drive the enhancer activity in this region: Rarg (another retinoid-responsive factor), Myc, and Foxk1. Taken together, these combined analyses identified a number of candidate factors that could influence the enhancer activity identified in the Peril locus, and pose multiple interesting directions for future study into exactly how this gene body enhancer regulates target gene expression.

\section{Chromosome conformation capture reveals altered interactions with regulatory targets in Peril knockout mESCs}

To assess how Peril ablation affects regulatory interactions and higher-order chromatin conformation changes, we performed high-throughput chromosome conformation capture (Hi-C) on WT and Peril KO mESC (Fig. 4a, Additional file 3: Figure S4A), [32, 33]. Briefly, two replicates of WT and KO mESC cells were sequenced to an average depth of approximately 155 million combined reads and analyzed using the HiC-Pro pipeline [34]. Both sets of replicates showed high reproducibility (Additional file 3: Figure S4B-C), and reads across the Peril locus confirmed WT and KO genotypes (Additional file 3: Figure S4D).

The TAD structure surrounding the Peril locus consists of a series of small sub-TADs contained within a $2-\mathrm{Mb}$ TAD, while the two adjacent TADs $(-1$ TAD and +1 TAD) are smaller in size: $\sim 900 \mathrm{~kb}$ and $\sim 1 \mathrm{Mb}$, respectively (Fig. 4a). The 2-Mb Peril-containing TAD contains only a few genes and one centrally located sub-TAD, only $\sim 150 \mathrm{~kb}$ in size, which contains Peril, the super-enhancer, and Sox2. Interestingly, we found that Peril deletion does not lead to gross chromatin conformation changes on chromosome 3 (Fig. 4a), nor does it disrupt the $\sim 150-\mathrm{kb}$ sub-TAD containing Peril and Sox2. Thus, despite the association of Peril with Sox 2 within this sub-TAD, removal of DNA elements contained in the deleted region do not influence local architecture around the Sox2 locus. This is consistent with our finding that there is no significant expression effect on Sox 2 in any of the Peril knockout tissues and cell lines tested (Fig. 3). Thus, the majority of the Peril gene body is dispensable for Sox2 regulation.

To identify which regions on chromosome 3 interact specifically with the deleted region, we generated a custom mm10 genome build which replaces the deleted region with spacer nucleotides (Ns) and contains two separate chromosomes: one containing the deleted region sequence and one containing the $L a c Z$ sequence (Fig. 4b). We sought to perform a digital 4C-like analysis anchoring on the "deleted region" chromosome or "LacZ" chromosome in WT or KO samples, respectively, but were limited by the lack of restriction enzyme sites in the LacZ sequence (see the "Methods" section). Unable to directly compare the deleted region to the LacZ bait, we instead compared interactions from the 3' neighboring $20-\mathrm{kb}$ bin and found a significant decrease in interactions between WT and KO with the +1 TAD (see the "Methods" section, Fig. 4c, Additional file 3: Figure S4E). This indicates that, in the KO, these interactions with the neighboring regions are altered (Fig. 4c, Additional file 3: Figure S4E).

It has previously been shown that expression of genes within a TAD are correlated and that while TAD boundaries tend to insulate interactions between neighboring TADs, inter-TAD interactions do occur [35-40]. It is thus quite interesting to note that both $M c c c 1$ and Exosc9 are located within the +1 TAD relative to Peril. To gain insight into potential long-range regulatory effects of Peril deletion, we generated digital 4C-like plots anchored on either Mccc1 or Exosc9. Indeed, upon loss of Peril, we observe an alteration of contacts between these loci and the region just upstream of the Peril deletion (Fig. 4d). Specifically, Mccc1 appears to lose interactions with this region in $\mathrm{KO}$ cells, while the Exosc9 region appears to demonstrate a subtle gain of interactions. Thus, in our RNA-seq dataset, Mccc1 and Exosc 9 are transcriptionally downregulated in all tissues and cells, and in our $\mathrm{Hi}-\mathrm{C}$ dataset, these targets appear to show altered contacts with Peril. This, in combination with the decrease in Pol II binding at the target promoters in Peril knockout mESC, suggests that Mccc1 and Exosc9 are the targets of Peril DNA enhancers.

Together, our results indicate that the cis-regulatory elements at the Peril locus show altered interactions with the +1 TAD, which harbors the two consistently dysregulated genes, Mccc1 and Exosc9. These results provide evidence for enhancer elements encoded in the Peril locus that play a role in the regulation of $M c c c 1$ and Exosc9 expression. Ultimately, these data highlight the importance of considering DNA regulatory roles in examining any given genic locus.

\section{Discussion}

Recently, it has been shown that lincRNA loci influence the expression of their neighboring protein-coding genes [7, 22, 41-43]. However, the mechanisms of this regulation are largely uncharacterized. Here, we selected candidate lincRNAs based on their potential cis-regulatory function and directly tested the enhancer capacity of their loci at 50-bp resolution using MPRA technology. 


\section{A}

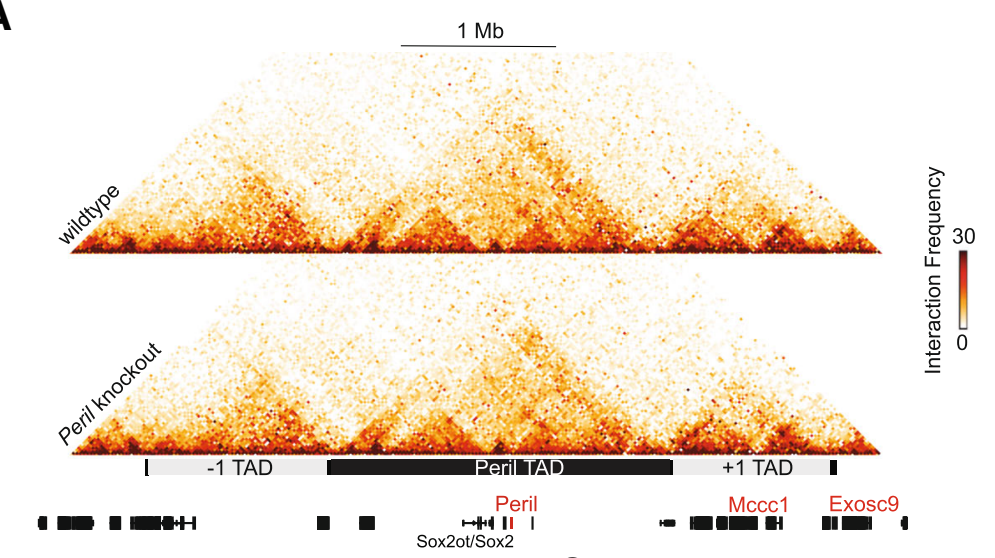

B

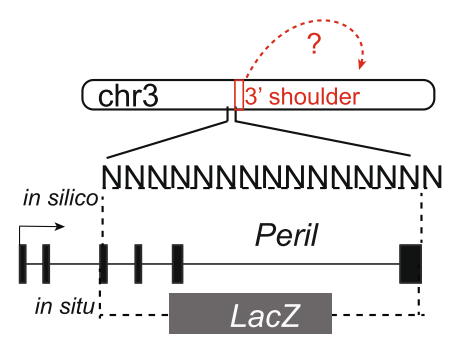

C
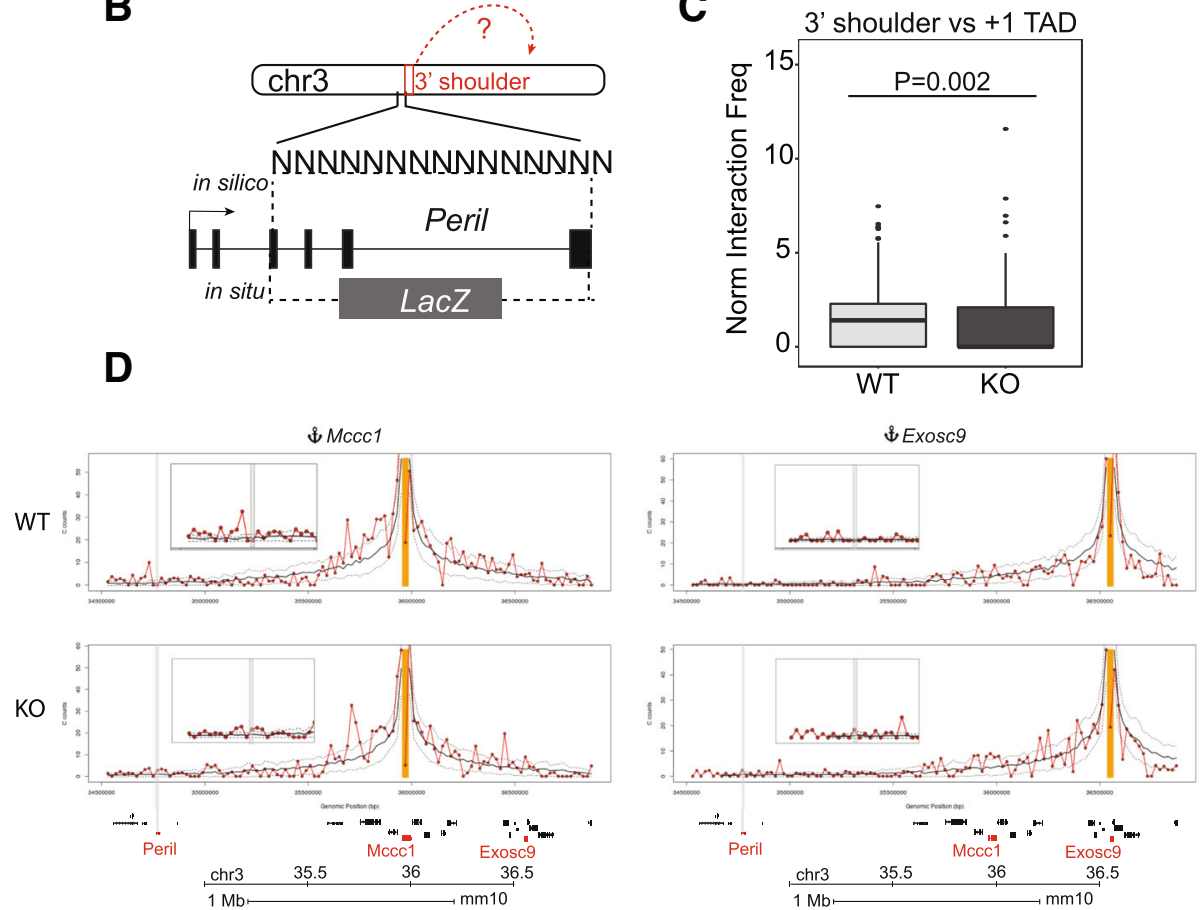

Fig. $4 \mathrm{Hi}-\mathrm{C}$ analysis of WT-v-Peril KO mESC. a Chromatin contact map surrounding the Peril locus at 20-kb resolution using custom mm10 genome build in wild-type (top) and knockout (bottom). Genes indicated below, with genes of interest indicated in red. TAD borders indicated in black; -1 and + 1 TADs demarcated by gray box while Peril-containing TAD is demarcated by a black box. b Mapping strategy of the Hi-C data to a custom mm10 mouse genome, where the deleted Peril region ( 14.5 kb) is replaced by Ns and this sequence or the LacZ ( 3.5 kb) are compiled as separate chromosomes. $3^{\prime}$ shoulder region (not affected by deletion, but used as a bait region common to both WT and KO conditions) indicated in red. c Digital 4C summary of normalized interactions between the 3' shoulder region (chr3:34784809-34800385) and the + 1 TAD in either WT or KO. d Digital 4C plots of the interaction anchored on either Mcccl or Exosc9 in WT and Peril KO Hi-C samples. The red line indicates the distancenormalized average interaction frequency, whereas the dashed lines represent the \pm 1 standard deviation. The 20-kb bins that are above the +1 standard deviation line are considered as enriched interactions. The red arrows indicate the alterations of long-range interactions of Mcccl and Exosc 9 with the Peril locus between WT and KO samples

Since we sought to test whether or not lincRNA loci would contain more enhancers or more highly active enhancers than protein-coding gene loci, we quantitatively mapped the enhancer capacity of a set of candidate cis-functional lincRNAs and their closest protein-coding neighbors.

Recent studies have suggested that promoters may play a larger role in cis-regulatory dynamics than previously appreciated [8-12]. Specifically, promoters are capable of exerting cis-regulatory function on neighboring genes in addition to promoting expression of the immediately adjacent sequence. Consistent with these studies, we found that indeed, the most enhancer activity within a locus, whether lincRNA or mRNA, occurs within the region $1 \mathrm{~kb}$ upstream of a TSS. However, we also identified DNA 
regulatory elements with varying activity levels within gene bodies in both mRNAs and lincRNAs. By combining a reporter knockout mouse model, in which the gene body but not promoter has been removed, with an MPRA (employed as a high-resolution enhancer mapping technique), we are able to identify novel enhancers as well as their candidate target genes. Together, these results underscore the importance of gene body DNA regulatory elements contained in both lincRNA and mRNA loci.

Resolving the targets of these DNA regulatory elements is nontrivial. To untangle modalities of regulation at lincRNA loci and to identify potential gene targets, we used the Peril locus as a model. The Peril locus overlaps with a Sox2 super-enhancer and forms a tightly associated sub-TAD with the Sox2 locus (Fig. 4b, [23, 25]). It also generates a spliced lincRNA and, by $L a c Z$ reporter expression, appears to be active in tissues that express Sox2 $[6,21,44]$. These findings all suggest a connection between Peril and Sox2. However, using unbiased RNA-seq approaches in vivo, we found no significant evidence for Peril regulation of Sox2. Instead, we discovered consistent and significant downregulation of two genes located within the neighboring TAD: Exosc9 and Mccc1. This method of using RNA sequencing across a variety of tissues to identify consistently misregulated genes, particularly in tissues where the lincRNA is not transcribed, has been used previously to identify the targets of DNA-based enhancer elements [3].

Comparison between wild-type and Peril knockout $\mathrm{Hi}-\mathrm{C}$ samples reveals alterations in interactions between the Peril region and the TAD containing the candidate target genes. It has been previously established that transcriptional co-regulation of genes is strongly associated with TAD formation $[35,40]$ and that regulatory modules can affect TAD-wide gene expression [37, 39]. The observation that Peril shows altered interaction frequency with target genes at the +1 TAD, (Figs. 3e and $4 \mathrm{c}$, d, Additional file 3: Figure S4E) suggests a possible functional role for Peril in the transcriptional regulation of these genes by long-range chromatin interactions. In part, this could occur by DNA elements within the Peril locus influencing Pol II occupancy at the Mccc1 and Exosc9 promoters, which is supported by our ChIP-qPCR data (Additional file 3: Figure S3D). Yet, the exact mechanisms of the establishment and maintenance of these long-range interactions remain to be determined. Some insight is provided by our intersection of publicly available ChIP-seq data with the Peril enhancer region identified by MPRA, in which we have identified potential binding of important genome organizer factors such as Rad21 and Ctcf, as well as Rxra. All of these factors have been significantly implicated in chromatin looping and enhancer function $[29,45,46]$.
Consistent with our previous report of perinatal lethality among Peril knockout mice [21], loss of Mccc1 and Exosc9 are documented to have deleterious effects in human and mouse, respectively, including prenatal lethality [47-50]. We find that both $M c c c 1$ and Exosc 9 are consistently and specifically downregulated as a consequence of Peril ablation. Consistent with Peril targeting Mccc1 and Exosc9, we observed fewer physical interactions exist between the Peril locus and the TAD containing these genes in knockout mESC. These data point to a potential in vivo DNA-based mechanism for Peril: function through control of distally located, developmentally important genes. Although we can rule out the role of the Peril RNA in regulating some target genes (e.g., Mccc1 and Exos(9), we cannot exclude an additional role for the RNA in trans. Consistent with this possibility, we observed numerous gene expression changes in the Peril knockout mESC line which could be due to loss of the RNA product, though this effect could also be due to a cell type-specific effect of reduced Mccc1 or Exosc9 transcript levels.

Beyond Peril, our study further highlights the DNA regulatory potential contained in promoters and gene bodies regardless of coding classification (i.e., lincRNA or mRNA). We found that both lincRNAs and mRNAs contain cis-regulatory potential in their promoters, as described previously $[8-12,15]$. Furthermore, we demonstrate that in both gene types, regulatory potential is additionally contained in gene bodies, though we found that high-activity regions occurred in more of the lincRNA (Lincp21, LincEnc1, and Peril) than mRNA (Morc2a) gene bodies we queried.

The presence of clear enhancer activity in these loci has important implications for our current understanding and interpretation of lincRNA knockout models, which have been the focus of intense debate [5, 22]. Specifically, these results highlight the potential for a given locus to generate a spliced gene product and also contain functional DNA elements. This work and others $[3,4]$ highlight that the functions of these two molecular species should not be conflated, but rather independently assessed. It should also be noted that mRNA gene bodies have been known to harbor enhancers as well, as established by numerous ChIP-seq experiments, or conservation mapping [13, 51]. For example, Dync1l1 (an mRNA locus) harbors exonic enhancers which regulate nearby genes $D l \times 5 / 6$, and removal of these regulatory sequences can lead to disease [18]. Similarly, an intronic sequence in $L m b r 1$ acts over 1 $\mathrm{Mb}$ to regulate Shh expression in the developing limb [51]. Currently, hundreds of knockout strains and ESC lines targeting mRNA loci have been generated using whole gene ablation techniques (for example, by the immense efforts of the Knockout Mouse Project 
(KOMP) and the International Mouse Phenotyping Consortium (IMPC)) [50, 52-54]. Some of these strains use the same whole locus gene targeting technique as our lincRNA mouse models, which means that the same complications will arise in resolving the functional molecular species at these mRNA loci. Here, we provide a logical framework for follow-up studies in these models to identify DNA elements and their regulatory targets.

\section{Conclusions}

Here, we conclude, by combining MPRA, in vivo deletion/reporter models, and $\mathrm{Hi}-\mathrm{C}$, that both mRNAs and lincRNAs can contain DNA regulatory elements in their gene bodies. Further investigation of one of these DNA elements in vivo revealed a surprising finding: that Peril regulates two distally located genes (Mccc1 and Exosc9) and not its neighbor Sox2. Despite close proximity of the Peril locus with Sox2, we find that neither the DNA nor the RNA in the Peril locus affect Sox 2 expression. Moreover, we find that ablation of Peril does not affect the local chromatin architecture, but does affect long-range interactions with $M c c c 1$ and Exosc9 in the neighboring TAD. Together, our combined approach is able to identify DNA regulatory elements in lincRNAs and their candidate regulatory targets in vivo.

\section{Methods}

\section{Massively parallel reporter assay}

The MRPA pool was designed as previously described $[3,55]$, with the following important alterations. (1) The full gene body of each locus and $1000 \mathrm{bp}$ upstream (the promoter region) were included in the design. (2) Five micrograms of GFP+ plasmid was used to transfect $\mathrm{C} 2 \mathrm{C} 12$ cells in triplicate wells of six-well dishes using lipofectamine 2000 (Thermo Fisher 11668027) and lowserum, antibiotic-free media. RNA was harvested using TRIzol, and libraries were generated as described previously $[3,55]$. Five replicate samples were generated for RNA samples (taken from biological replicates at two different passages), as for DNA samples (generated on two separate days). Libraries were generated via PCR with indexed primers. For RNA libraries, $1 \mu \mathrm{g}$ RNA input was used, while for DNA libraries, $1 \mathrm{ng}$ of the MPRA vector was used. Samples were sequenced on a Hiseq 2500 at Harvard University's Bauer Sequencing Core.

\section{C2C12 culture}

C2C12 cells were acquired from ATCC (CRL-1772) and cultured in DMEM supplemented with FBS and antibiotics.

\section{MPRA analysis overview}

Analysis was performed largely as described previously [3] with three important differences in the calculation of the activity ratio between RNA and DNA replicates. (1) After normalizing for sequencing depth, each sample was median-normalized. (2) The activity ratio for each barcode was determined by randomly selecting one RNA replicate and one DNA replicate and calculating the ratio normed_RNA/normed_DNA. This process was repeated 1000 times, and the resulting median was reported as the final signal ratio for that barcode. (3) Calculation of significant regions was performed by shuffling sample labels rather than barcode labels. In other words, we repeated step 2 without labeling which samples were RNA or DNA to generate a null distribution against which to compare true signal in any given region. $p$ values were corrected using the Benjamini-Hochberg method with an FDR of $1 \%$. All analysis was performed using the aid of in-house python and $\mathrm{R}$ scripts, with aid from biopython and R's bioconductor packages. Oligo annotation is available as Additional file 1, TSS annotation is available as Additional file 2, and all code is available as Additional file 4. Additionally, code is available under the MIT License on github (https://github. com/agroff11/PerilPaperAnalysis) and on zenodo (https:// doi.org/10.5281/zenodo.1477113) [56].

\section{Mice}

Mice were housed under pathogen-free conditions at Harvard University. A description of the mice used in this experiment is available as Additional file 5. The Peril knockout strain was originally generated by Regeneron Pharmaceuticals [21, 57].

\section{Derivation and culture of $\mathrm{mESC}$}

Wild-type and Peril knockout murine ESCs were generated by the Harvard University Genome Modification Facility from D3.5 embryos. After receiving clones from the core, mESC colonies were transitioned to $2 \mathrm{i}$ on feeder MEFs and cultured for six passages. Three individual wells (derived from the same original clone) were collected as biological replicates.

\section{Tissue collection}

Three biological replicates of the kidney and two biological replicates of microdissected ganglionic eminence and olfactory bulb (GE/OB) were harvested from E14.5 progeny of heterozygous matings. Tissues were pulverized in TRIzol and snap frozen.

\section{RNA library generation and sequencing}

The Illumina TruSeq kit was used to create polyA ${ }^{+}$libraries from $250 \mathrm{ng}$ total RNA for each tissue replicate and $500 \mathrm{ng}$ total RNA for each mESC replicate. Samples were sequenced on a Hiseq 2500 at Harvard University's Bauer Sequencing Core. 


\section{Sequence alignment and analysis}

Reads were aligned to $\mathrm{mm} 10$ and quantified using rsem with nonstandard parameters --paired-end --bowtie2 --append-names and -p 8 [58]. Read counts from this quantification were used in downstream differential expression analysis using DESeq (alpha $=0.05$ for all comparisons, [59]). Reads in the knocked out region of Peril were assessed visually to confirm knockout status in combination with expression status of LacZ. All RNAseq figures were generated in $\mathrm{R}$ with the help of packages available on bioconductor. Code is available as Additional file 6 and is available under the MIT license on github (https://github. com/agroff11/PerilPaperAnalysis) and on zenodo (DOI: https://doi.org/10.5281/zenodo.1477113) [56].

\section{qRT-PCR}

cDNA was generated using SuperScriptIII (Thermo Fisher), and qRT-PCR analyses were performed using an ABI7900HT Fast Real-Time PCR system (applied biosystems). Data was analyzed using qPCR-miner [60], and the ddCT method was used to calculate relative cDNA. Samples were normalized to L32 control. Primers used are as follows: Mccc1 (L) gggtgtgcagtcggtggctg, Mccc1 (R) gcctggatggatggcctgtgc, Exosc9 (L) aagagcgtgatcccgtgcca, Exosc9 (R) tggcaatcaccagcaagccatcc, L32 (L): aacccagaggcattgacaac, L32 (R): attgtggaccaggaacttgc.

\section{Transcription factor binding analysis}

We downloaded the entire set of motifs available through JASPAR (the JASPAR vertebrate collection, [30]) and filtered these for an average expression greater than or equal to 1TPM in the tissues and cells used in this paper. We then queried the sequence of the high-activity peak in the Peril gene body for matches to any of the expressed transcription factors on a per-sample-type basis using FIMO, a motif-finding tool publically available through the meme suite [31]. We filtered sequence matches for those with a $q$ value $<0.05$ and plotted the binding profiles of the common set of expressed hits. Additionally, we assessed publically available TF binding via the ChIP-atlas [61].

\section{Chromatin immunoprecipitation (ChIP)}

ChIP experiments were performed using the iDeal ChIP-qPCR kit (Diagenode, C01010180), and experiments were carried out according to the manufacture protocol. Briefly, approximately 16 million wild-type or 16 million Peril knockout mESC were crosslinked for 10 min in 1\% formaldehyde. Chromatin was sheared with the following parameters: $15 \mathrm{~min}$ of $30 \mathrm{~s}$ "on" and $30 \mathrm{~s}$ "off" using the Bioruptor (Diagenode). ChIP experiments were performed using $2 \mu \mathrm{L}$ of Pol II monoclonal antibody (Diagenode, C15100055) per ChIP, and as negative control, $1 \mu \mathrm{g}$ of IgG was used in a separate ChIP. For downstream enrichment calculation, $1 \%$ of the input chromatin was retained. The occupancy of Pol II at the promoter of Mccc1 and Exosc9 was measured by real-time qPCR using two primer sets targeting different regions of the promoters. qPCR was performed using FastStart Universal SYBR Green Master (Rox) (ABI, 4913914001), and reactions were run on ViiA7 Real-Time PCR System (ABI). Primer sets used in the experiment targeted Pol II-enriched regions near the target gene promoters as well as control regions: F1_Mccc1: CAAG TAGGCGTCTCCGCAA; R1_Mccc1: GGTCCAATCAGC CAATCGGTA; F2_Mccc1: ATTCTTGAAAGCGTCCC TCCC; R2_Mccc1: CTTGCGGAGACGCCTACTTG; F3 Exosc9: ACATTGTCAGTTCGGCCTGT; R3_Exosc9: AA TACTGCCCTGAGGCTTGG; F4_Exosc9: GTGTGGAC TCTCCCCATTCC; R4_Exosc9: TCCCTGCCACTGAA TACTGC; F_Pol2_positive_region: CTGGCACTGCACA AGAAG; R_Pol2_positive_region: GGGTTCCTATAAAT ACGG; F_Pol2_negative_region: CTGGCCTCCATACA CACATA; R_Pol2_negative_region: AGTCAGCAGGAT CCACACTT. Enrichment was calculated by the percent input method.

\section{Generation and analysis of $\mathrm{Hi}-\mathrm{C}$ libraries}

In situ Hi-C libraries were generated using the HindIII restriction enzyme $[32,33]$. Briefly, $\sim 25$ million cells were crosslinked with $1 \%$ formaldehyde for $10 \mathrm{~min}$ at room temperature. Then, the chromatin was extracted, digested with HindIII, end-labeled with biotin-14-dCTP, followed by an in situ ligation procedure. After DNA extraction, biotin was removed from unligated ends, and the sample was sheared by using a Covaris S220 instrument (100-500 bp range). After A-tailing, biotin pull-down and adapter ligation, paired-end sequencing was performed on a HiSeq instrument. Each $\mathrm{Hi}-\mathrm{C}$ library was generated in two biological replicates, and each replicate was sequenced to a depth of $\sim 80$ million reads. Hi-C mapping, filtering, iterative correction, and binning were performed with the HiC-Pro software v2.8 [34]. We used multiple genome builds in our analyses: for general quality control and structural analyses, we used both $\mathrm{mm} 9$ and $\mathrm{mm} 10$, and for the digital $4 \mathrm{C}$ analyses, we used a custom mm10 genome which replaced the deleted Peril region with Ns and then included this sequence as well as the LacZ reporter sequence as two separate chromosomes.

There was a high correlation among the Hi-C biological replicates, and so we pooled all biological replicates for each condition and analyzed them as a single $\mathrm{Hi}-\mathrm{C}$ dataset. This pooled dataset was used for all the analyses. The TAD analysis was performed with the insulation method, as previously described [62], by using the cworld package (https://github.com/dekkerlab/cworld-dekker) [63] "matrix2insulation.pl" script with the following options: -is 480000 -ids 320000 -im iqrMean -nt 0 -ss 160000 -yb 
1.5 -bmoe 0 -bg. The digital 4C plots were generated with the cworld package "matrix2anchorplot.pl" script and with the HiC-Pro "make_viewpoints.py" script by using either the Mccc1 or the Exosc9 loci as anchor points. To do this, we calculated the $\mathrm{Hi}-\mathrm{C}$ interaction frequency between the Mccc1/Exosc9-containing bins and the neighboring 20-kb bins. WT interactions were mapped to the $\mathrm{mm} 10$ reference genome while $\mathrm{KO}$ interactions were mapped to our custom mm10 genome build. For each distance, the \pm 1 standard deviation of all the $\mathrm{Hi}-\mathrm{C}$ interaction frequencies of that distance (dashed lines in the figure) were also calculated. The interactions that were above the +1 standard deviation line (i.e., positive $z$-score) were considered as enriched interactions. To investigate the interactions between the Peril region and the neighboring TAD, we first investigated the HindIII restriction sites in the deleted region or LacZ "chromosomes." We found that the "deleted region chromosome" contains three cut sites while the "LacZ chromosome" contains none. We thus selected the 3' shoulder region (encompassing the 6 downstream HindIII sites, $\sim 15 \mathrm{~kb}$, chr3:34784809-34800385). This region is shared between WT and KO and is not affected by the deletion. While not specific to the deleted sequence, it can be used as a proxy for alterations in interactions between $\mathrm{WT}$ and $\mathrm{KO}$ in the deleted region. To generate digital 4C-like bedgraphs anchored on this shoulder region, we plotted either raw reads originating in this region and ending on chromosome 3, or a boxplot of all normalized reads originating from this region and ending in the +1 TAD. The boxplot was generated using the custom genome for both WT and KO samples.

\section{Additional files}

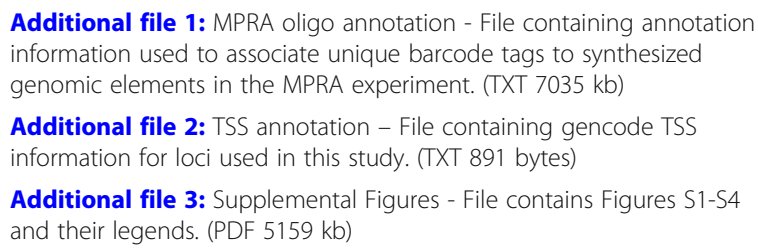

Additional file 4: MPRA analysis code - $R$ markdown file containing all code used in the MPRA analysis. (RMD $38 \mathrm{~kb}$ )

Additional file 5: Mouse master sheet - File containing description of mice used in this study, and information about generation of RNA sequencing libraries. (XLSX $52 \mathrm{~kb}$ )

Additional file 6: RNA seq analysis code - $\mathrm{R}$ markdown file containing all code used in the RNA-sequencing analysis. (RMD $15 \mathrm{~kb}$ )

\section{Acknowledgements}

We thank all members of the Rinn Lab for the critical evaluation of this work and particularly Chiara Gerhardinger for her help with the RNA sequencing data. We would also like to thank Rafael Irizarry for helpful discussions related to the MPRA analysis. Additionally, we thank James Lee and Chiara Gerhardinger for feedback on the manuscript, as well as Martin Sauvageau for his contributions to establishing the mouse knockout strains in the lab.

\section{Funding}

AG is supported by HHMI as a Gilliam Fellow. JR is the Leslie Orgel Professor for RNA Biology and Marvin Caruthers chaired professor. This work was funded by P01GM099117, R01MH102416, and HHMI Faculty Scholars Program.

\section{Availability of data and materials}

All sequencing files (MPRA, RNAseq, and $\mathrm{Hi}-\mathrm{C}$ ) are deposited in GEO with the accession number GSE104341 [64]. Accession numbers for other RNA-seq datasets used in this analysis are GSE61716 (whole brain samples, [65]) and GSE20846 (C2C12 RNA sequencing [66]). All code is available under the MIT license on github (https://github.com/agroff11/PerilPaperAnalysis) and on zenodo (DOI: https://doi.org/10.5281/zenodo.1477113, [56]). Additionally, we used Hi-C packages from https://github.com/dekkerlab/cworld-dekker [63] and https://github.com/nservant/HiC-Pro [34].

\section{Authors' contributions}

AFG and JLR conceived the study and designed the experiments. ARB performed the $\mathrm{Hi}-\mathrm{C}$ experiments and analysis. JPL performed the Pol II ChIP and analysis. AFG carried out all other experiments and analyzed the data with input from JLR. AFG, $A R B, J P L$, and JLR wrote the manuscript. All authors read and approved of the final manuscript.

\section{Ethics approval and consent to participate}

Procedures were all approved by the Harvard University Committee on the Use of Animals in Research and Teaching and performed in accordance with the National Institutes of Health guidelines.

\section{Consent for publication}

Not applicable

\section{Competing interests}

The authors declare that they have no competing interests.

\section{Publisher's Note}

Springer Nature remains neutral with regard to jurisdictional claims in published maps and institutional affiliations.

\section{Author details}

'Department of Stem Cell and Regenerative Biology, Harvard University, Cambridge, MA 02138, USA. ²Department of Systems Biology, Harvard Medical School, Boston, MA 02115, USA. ${ }^{3}$ Donnelly Centre, University of Toronto, Toronto, ON M5S 3E1, Canada. ${ }^{4}$ Department of Biochemistry, BioFrontiers, University of Colorado Boulder, Boulder, CO 80301, USA.

Received: 6 February 2018 Accepted: 16 November 2018

Published online: 11 December 2018

References

1. Wang KC, Chang HY. Molecular mechanisms of long noncoding RNAs. Mol Cell. 2011;43:904-14

2. Yin Y, Yan P, Lu J, Song G, Zhu Y, Li Z, et al. Opposing roles for the IncRNA haunt and its genomic locus in regulating HOXA gene activation during embryonic stem cell differentiation. Cell Stem Cell. 2015;16:504-16.

3. Groff AF, Sanchez-Gomez DB, Soruco MML, Gerhardinger C, Barutcu AR, Li E, et al. In vivo characterization of Linc-p21 reveals functional cis-regulatory DNA elements. Cell Rep. 2016;16:2178-86.

4. Paralkar VR, Taborda CC, Huang P, Yao Y, Kossenkov AV, Prasad R, et al. Unlinking an IncRNA from its associated cis element. Mol Cell. 2016;62:104-10.

5. Bassett AR, Akhtar A, Barlow DP, Bird AP, Brockdorff N, Duboule D, et al. Considerations when investigating IncRNA function in vivo. Elife. 2014;3: e03058.

6. Goff LA, Groff AF, Sauvageau M, Trayes-Gibson Z, Sanchez-Gomez DB, Morse $\mathrm{M}$, et al. Spatiotemporal expression and transcriptional perturbations by long noncoding RNAs in the mouse brain. Proc Natl Acad Sci U S A. 2015; 112:6855-62.

7. Kopp F, Mendell JT. Functional classification and experimental dissection of long noncoding RNAs. Cell. 2018;172:393-407.

8. Arnold CD, Zabidi MA, Pagani M, Rath M, Schernhuber K, Kazmar T, et al. Genome-wide assessment of sequence-intrinsic enhancer responsiveness at single-base-pair resolution. Nat Biotechnol. 2017;35:136-44. 
9. Sanyal A, Lajoie BR, Jain G, Dekker J. The long-range interaction landscape of gene promoters. Nature. 2012;489:109-13.

10. Dao LTM, Galindo-Albarrán AO, Castro-Mondragon JA, Andrieu-Soler C, Medina-Rivera A, Souaid C, et al. Genome-wide characterization of mammalian promoters with distal enhancer functions. Nat Genet. 2017;49:1073-81.

11. Freire-Pritchett P, Schoenfelder S, Várnai C, Wingett SW, Cairns J, Collier AJ, et al. Global reorganisation of cis-regulatory units upon lineage commitment of human embryonic stem cells. Elife. 2017;6:699.

12. Sahlén P, Abdullayev I, Ramsköld D, Matskova L, Rilakovic N, Lötstedt B, et al. Genome-wide mapping of promoter-anchored interactions with close to single-enhancer resolution. Genome Biol. 2015;16:156.

13. Visel A, Blow MJ, Li Z, Zhang T, Akiyama JA, Holt A, et al. ChIP-seq accurately predicts tissue-specific activity of enhancers. Nature. 2009;457:854-8.

14. ENCODE Project Consortium. An integrated encyclopedia of DNA elements in the human genome. Nature. 2012:489:57-74.

15. Engreitz JM, Haines JE, Perez EM, Munson G, Chen J, Kane M, et al. Local regulation of gene expression by IncRNA promoters, transcription and splicing. Nature. 2016;539:452-5.

16. Meng F-L, Du Z, Federation A, Hu J, Wang Q, Kieffer-Kwon K-R, et al. Convergent transcription at intragenic super-enhancers targets AID-initiated genomic instability. Cell. 2014;159:1538-48.

17. Kowalczyk MS, Hughes JR, Garrick D, Lynch MD, Sharpe JA, Sloane-Stanley JA, et al. Intragenic enhancers act as alternative promoters. Mol Cell. 2012:45:447-58.

18. Birnbaum RY, Clowney EJ, Agamy O, Kim MJ, Zhao J, Yamanaka T, et al. Coding exons function as tissue-specific enhancers of nearby genes. Genet Res. 2012;22:1059-68.

19. González-Vallinas J, Pagès A, Singh B, Eyras E. A semi-supervised approach uncovers thousands of intragenic enhancers differentially activated in human cells. BMC Genomics. 2015;16:523.

20. Ritter DI, Dong Z, Guo S, Chuang JH. Transcriptional enhancers in proteincoding exons of vertebrate developmental genes. Schubert M, editor. PLOS ONE. 2012;7:e35202.

21. Sauvageau M, Goff LA, Lodato S, Bonev B, Groff AF, Gerhardinger C, et al. Multiple knockout mouse models reveal lincRNAs are required for life and brain development. Elife. 2013;2:e01749.

22. Goff LA, Rinn JL. Linking RNA biology to IncRNAs. Genome Res. 2015;25: 1456-65.

23. Li Y, Rivera CM, Ishii H, Jin F, Selvaraj S, Lee AY, et al. CRISPR reveals a distal super-enhancer required for Sox2 expression in mouse embryonic stem cells. Wu Q, editor. PLoS One; 2014;9:e114485.

24. Zhang S, Cui W. Sox2, a key factor in the regulation of pluripotency and neural differentiation. World I Stem Cells. 2014;6:305-11.

25. Zhou HY, Katsman Y, Dhaliwal NK, Davidson S, Macpherson NN, Sakthidevi $M$, et al. A Sox2 distal enhancer cluster regulates embryonic stem cell differentiation potential. Genes Dev. 2014;28:2699-711.

26. Ernst J, Melnikov A, Zhang X, Wang L, Rogov P, Mikkelsen TS, et al. Genome-scale high-resolution mapping of activating and repressive nucleotides in regulatory regions. Nat Biotechnol. 2016;34:1180-90.

27. Kheradpour P, Ernst J, Melnikov A, Rogov P, Wang L, Zhang X, et al. Systematic dissection of regulatory motifs in 2000 predicted human enhancers using a massively parallel reporter assay. Genet Res. 2013;23:800-11.

28. Melnikov A, Zhang X, Rogov P, Wang L, Mikkelsen TS. Massively parallel reporter assays in cultured mammalian cells. J Vis Exp. 2014;(90). https://doi. org/10.3791/51719.

29. Daniel B, Nagy G, Hah N, Horvath A, Czimmerer Z, Poliska S, et al. The active enhancer network operated by liganded RXR supports angiogenic activity in macrophages. Genes Dev. 2014;28:1562-77.

30. Khan A, Fornes O, Stigliani A, Gheorghe M, Castro-Mondragon JA, van der Lee R, et al. JASPAR 2018: update of the open-access database of transcription factor binding profiles and its web framework. Nucleic Acids Res. 2018;46:D1284.

31. Grant CE, Bailey TL, Noble WS. FIMO: scanning for occurrences of a given motif. Bioinformatics (Oxford, England). 2011;27:1017-8.

32. Belton J-M, McCord RP, Gibcus JH, Naumova N, Zhan Y, Dekker J. Hi-C: a comprehensive technique to capture the conformation of genomes. Methods. 2012;58:268-76.

33. Belaghzal H, Dekker J, Gibcus JH. Hi-C 2.0: an optimized Hi-C procedure for high-resolution genome-wide mapping of chromosome conformation. Methods. 2017;123:56-65.

34. Servant N, Varoquaux N, Lajoie BR, Viara E, Chen C-J, Vert J-P, et al. HiC-Pro: an optimized and flexible pipeline for $\mathrm{Hi}-\mathrm{C}$ data processing. Genome Biol. 2015;16:11 Available from: https://github.com/nservant/HiC-Pro.
35. Dixon JR, Selvaraj S, Yue F, Kim A, Li Y, Shen Y, et al. Topological domains in mammalian genomes identified by analysis of chromatin interactions. Nature. 2012;485:376-80.

36. Le Dily F, Baù D, Pohl A, Vicent GP, Serra F, Soronellas D, et al. Distinct structural transitions of chromatin topological domains correlate with coordinated hormone-induced gene regulation. Genes Dev. 2014;28:2151-62.

37. Symmons O, Uslu W, Tsujimura T, Ruf S, Nassari S, Schwarzer W, et al. Functional and topological characteristics of mammalian regulatory domains. Genome Res. 2014;24:390-400.

38. Rao SSP, Huntley MH, Durand NC, Stamenova EK, Bochkov ID, Robinson JT, et al. A 3D map of the human genome at kilobase resolution reveals principles of chromatin looping. Cell. 2014;159:1665-80.

39. Smith EM, Lajoie BR, Jain G, Dekker J. Invariant TAD boundaries constrain cell-type-specific looping interactions between promoters and distal elements around the CFTR locus. Am J Hum Genet. 2016:98:185-201.

40. Lupiáñez DG, Kraft K, Heinrich V, Krawitz P, Brancati F, Klopocki E, et al. Disruptions of topological chromatin domains cause pathogenic rewiring of gene-enhancer interactions. Cell. 2015;161:1012-25.

41. Ørom UA, Derrien T, Beringer M, Gumireddy K, Gardini A, Bussotti G, et al. Long noncoding RNAs with enhancer-like function in human cells. Cell. 2010;143:46-58.

42. Cabili MN, Trapnell C, Goff L, Koziol M, Tazon-Vega B, Regev A, et al. Integrative annotation of human large intergenic noncoding RNAs reveals global properties and specific subclasses. Genes Dev. 2011;25:1915-27.

43. Guil S, Esteller M. Cis-acting noncoding RNAs: friends and foes. Nat Struct Mol Biol. 2012;19:1068-75.

44. Guttman M, Amit I, Garber M, French C, Lin MF, Feldser D, et al. Chromatin signature reveals over a thousand highly conserved large non-coding RNAs in mammals. Nature. 2009:458:223-7.

45. Guo Y, Xu Q, Canzio D, Shou J, Li J, Gorkin DU, et al. CRISPR inversion of CTCF sites alters genome topology and enhancer/promoter function. Cell. 2015;162:900-10.

46. Rao SSP, Huang S-C, Glenn St Hilaire B, Engreitz JM, Perez EM, KiefferKwon K-R, et al. Cohesin loss eliminates all loop domains. Cell. 2017;171: 305-24.

47. Rips J, Almashanu S, Mandel H, Josephsberg S, Lerman-Sagie T, Zerem A, et al. Primary and maternal 3-methylcrotonyl-CoA carboxylase deficiency: insights from the Israel newborn screening program. J Inherit Metab Dis. 2016;39:211-7.

48. Grünert SC, Stucki M, Morscher RJ, Suormala T, Bürer C, Burda P, et al. 3-methylcrotonyl-CoA carboxylase deficiency: clinical, biochemical, enzymatic and molecular studies in 88 individuals. Orphanet J Rare Dis. 2012;7:31.

49. Mukherjee D, Gao M, O'Connor JP, Raijmakers R, Pruijn G, Lutz CS, et al. The mammalian exosome mediates the efficient degradation of mRNAs that contain AU-rich elements. EMBO J. 2002;21:165-74.

50. Brown SDM, Moore MW. The International Mouse Phenotyping Consortium: past and future perspectives on mouse phenotyping. Mamm Genome. 2012;23:632-40.

51. Lettice LA, Heaney SJH, Purdie LA, Li L, de Beer P, Oostra BA, et al. A longrange Shh enhancer regulates expression in the developing limb and fin and is associated with preaxial polydactyly. Hum Mol Genet. 2003;12:1725-35.

52. International Mouse Knockout Consortium, Collins FS, Rossant J, Wurst W. A mouse for all reasons. Cell. 2007;128:9-13.

53. Skarnes WC, Rosen B, West AP, Koutsourakis M, Bushell W, lyer V, et al. A conditional knockout resource for the genome-wide study of mouse gene function. Nature. 2011;474:337-42.

54. Dickinson ME, Flenniken AM, Ji X, Teboul L, Wong MD, White JK, et al. Highthroughput discovery of novel developmental phenotypes. Nature. 2016; 537:508-14.

55. Melnikov A, Murugan A, Zhang X, Tesileanu T, Wang L, Rogov P, et al. Systematic dissection and optimization of inducible enhancers in human cells using a massively parallel reporter assay. Nat Biotechnol. 2012;30:271-7.

56. Enhancers in the Peril lincRNA locus regulate distant but not local genes [Internet]. github. Available from: https:/github.com/agroff11/PerilPaperAnalysis

57. Lai KM, Gong G, Atanasio A, Rojas J, Quispe J, Posca J, et al. Diverse phenotypes and specific transcription patterns in twenty mouse lines with ablated LincRNAs. PLoS One. 2015;10(4):e0125522.

58. Li B, Dewey CN. RSEM: accurate transcript quantification from RNA-Seq data with or without a reference genome. BMC Bioinformatics. 2011;12:323.

59. Love MI, Huber W. Anders S. Moderated estimation of fold change and dispersion for RNA-seq data with DESeq2. Genome Biol. 2014;15:550. 
60. Zhao S, Fernald RD. Comprehensive algorithm for quantitative real-time polymerase chain reaction. J Comput Biol. 2005;12:1047-64.

61. Oki S, Ohta T, Shioi G, Hatanaka H, Ogasawara O, Okuda Y, et al. ChIP-Atlas: a data-mining suite powered by full integration of public ChIP-seq data. EMBO Rep. 2018. https://doi.org/10.15252/embr.201846255. [Epub ahead of print].

62. Crane E, Bian Q, McCord RP, Lajoie BR, Wheeler BS, Ralston EJ, et al.

Condensin-driven remodelling of $X$ chromosome topology during dosage compensation. Nature. 2015;523:240-4.

63. Cworld [Internet]. github. Available from: https:/github.com/dekkerlab/ cworld-dekker. Accessed Dec 2018.

64. The Peril DNA locus acts as an in vivo enhancer for distal but not proximal genes [Internet]. GEO. Available from: https://www.ncbi.nlm.nih.gov/geo/ query/acc.cgi?acc=GSE104341

65. Transcriptional perturbations by long noncoding RNAs with distinct spatiotemporal expression in the mammalian brain [Internet]. GEO. Available from: https://www.ncbi.n/m.nih.gov/geo/query/acc.cgi?acc=GSE61716

66. Transcript assembly and abundance estimation from RNA-Seq reveals thousands of new transcripts and switching among isoforms [Internet]. GEO. Available from: https:/www.ncbi.nlm.nih.gov/geo/query/acc.cgi?acc=GSE20846. Accessed 3 Mar 2016.

Ready to submit your research? Choose BMC and benefit from:

- fast, convenient online submission

- thorough peer review by experienced researchers in your field

- rapid publication on acceptance

- support for research data, including large and complex data types

- gold Open Access which fosters wider collaboration and increased citations

- maximum visibility for your research: over $100 \mathrm{M}$ website views per year

At $\mathrm{BMC}$, research is always in progress.

Learn more biomedcentral.com/submissions 